\title{
Shaken Baby Syndrome: literature review in the last 5 years
}

\author{
Maycon Cristian Gomes De Paula1 ${ }^{1}$ Carlos Umberto Pereira², Nícollas Nunes Rabelo
}

Received: 21 April 2020 / Published: 01 August 2020

\begin{abstract}
Background: The aim of this study was to perform a narrative review on what the literature about shaken baby syndrome brought us in the last five years. Methods: A search on PubMed and Scielo using the keywords "Shaken Baby Syndrome", "Abusive Head Trauma" and "Síndrome do Bebê Sacudido" found 842 articles. After apply the inclusion and exclusion criteria we defined the sample corpus in 54 articles.

Results: In the last five years several medico-legal debates have taken place discussing the controversies in the pathophysiology and diagnosis of abusive head trauma. Despite this, it is still the most common cause of brain damage in child. Prevention methods were created and tested. Although some have been successful they need some adaptations to improve the effectiveness.

Conclusion: The Shaken Baby Syndrome is a high incidence disease that involves several neurological, cognitive and motor impairments. Because of that new research needs to be done to improve our knowledge about this type of trauma and the effectiveness of the prevention methods.
\end{abstract}

Keywords: Shaken baby syndrome, Abusive head trauma, Child abuse, Traumatic brain injury, Nonaccidental trauma, Shaking.

\footnotetext{
1 University of Sapucaí's Valley, Pouso Alegre, Minas Gerais, Brazil.

2 Department of Neurosurgery of FBHC and Neurosurgery Service, Aracaju, Sergipe, Brazil.

${ }^{3}$ Department of Neurosurgery, School of Medicine UniAtenas, Paracatu-MG, Brazil.
}

To whom correspondence should be addressed: Nícollas Nunes Rabelo, MD [E-mail: nicollasrabelo@hotmail.com]

Journal homepage: www.sbnped.com.br

\section{Introduction}

The Shaken Baby Syndrome (SBS) is the most common type of brain injury in child and implies high morbidity and mortality rates for the victims [1, 2]. It is a type of nonaccidental head trauma whose damage to the brain and associated structures is caused by the excessive and repetitive process of violent head acceleration-deceleration or impact during the child abuse [3].

In 2009, the committee on child abuse and neglect, from the American Association of Pediatrics, recommended the replacement of the term SBS by Abusive Head Trauma (AHT). Now, SBS is understood as a colloquial form to refer to AHT. The decision was made because of the fact that the AHT includes broader features, as direct head trauma, and not only the shaking movement. [3, 4].

In the West, nonaccidental head injuries, where the most frequent mechanism in this classification is the act of shaking the baby (SBS), have estimated incidence between 20 to 40 cases per 100,000 child under one-year-old. The tendency is that this incidence decreases as the age group increases [5]. The mortality rate is between $10 \%$ to $30 \%$ of the cases, and significant neurological damage is found in, at least, $50 \%$ of the survivors. It is estimated to be associated to $80 \%$ of the infants deaths caused by brain injury [2]. On the other hand, the estimated cost, according reported data, exceeds US $\$ 300,000$ and US $\$ 1,000,000$, each case, with medical and long-term management, respectively [6].

The diagnosis of $\mathrm{AHT}$ is made in the presence of three findings: subdural hematoma, retinal hemorrhage and cerebral edema (encephalopathy). Besides being potentially fatal, AHT can cause severe cognitive, visual and motor impairments in victims [4, 7]. Such signs, with or without indicatives of head or bone structures impact, and without severe trauma history are, not rarely, the clinical presentation of the disease [8]. However, once these signs are not pathognomonic [9], there is an extensive number of 
differential diagnoses that need to be excluded in order to confirm. This research, besides been extensive and detailed, involves legal issues, what makes this syndrome extremely controversial [1].

Because of all that controversy and legal issues, we can understand the need to review the state of the science in that area to perceive better the syndrome, identifying gaps in the medical knowledge and, by this way, guide future research. In this way, it will be possible to reduce insecurity in diagnosing and managing this type of trauma.

\section{Methods}

It is a literature review. In order to answer the question: "What does the specialized health literature of the last five years bring us about Shaken baby syndrome?" we accessed the data base PubMed and Scielo.

The advanced search involved the use of the keywords "Shaken baby syndrome", "Abusive head trauma" and "Síndrome do bebê sacudido". The process consisted in identification, record of studies, mapping and analysis.

The article data were selected using the inclusion criteria: original article, have complete absctract in data base, in English or Portuguese, whose the study accords this narrative review's interests. Besides that the article should be full in eletronic format and must have been published in the last five years- between december 23rd, 2014 and december 23rd, 2019. The exclusion criteria were: experience report and articles that, despite addressing interested topic, dont have it as the central object of study.

The flow diagram of search strategy and study selection is shown on figure 1 .

\section{Results and Discussion}

\section{Pathophysiology}

\section{Cerebral edema (encephalopathy)}

It is related to brain injury.

The ischemia, main lethality factor in these cases, can be consequence of the hypoperfusion due to the intracranial hypertension caused by shaking. It can be associated to apnea in cases of early faints or spinal cord injuries. On the other hand, it can be linked to seizures. In the most of cases, these processes occur mutually and are enhanced, showing why SBS is, even with less energy involved, as harmful as accidental traumas[1] .

\section{Subdural collections}

They are the most common findings on abusive head trauma. It can be uni or bilateral [3].
The shear and rotational forces, caused by acceleration-deacceleration movements, breaks partially or completely the small and medium caliber

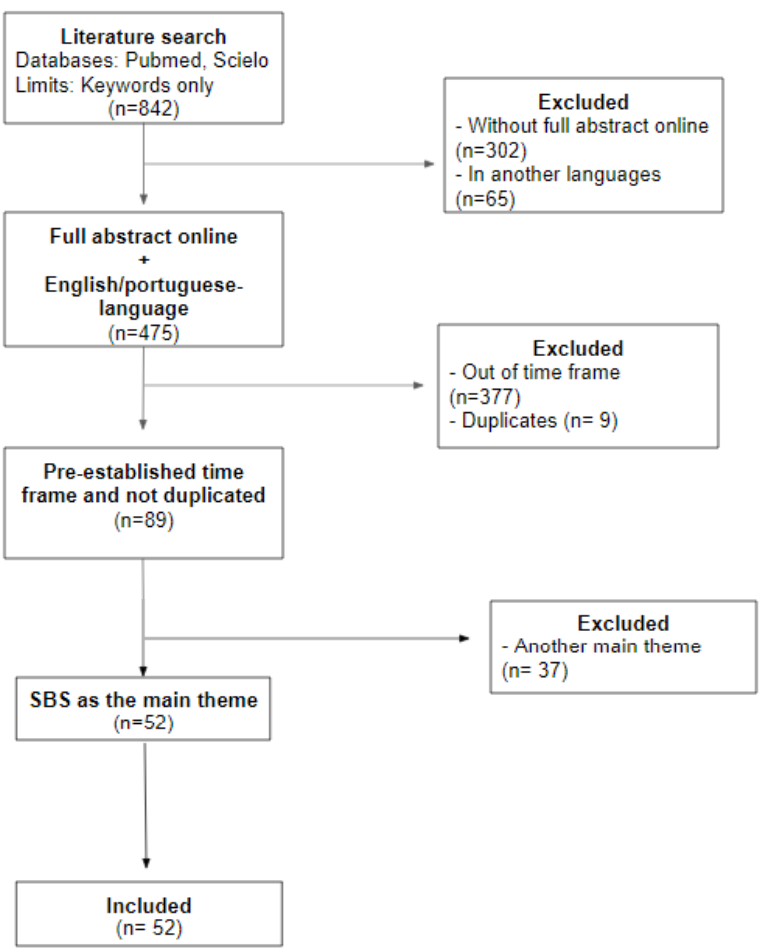

Figure 1: Flow diagram of search strategy and study selection

veins, amongst them the main ones, called bridgeveins. This rupture cause extra-axial hemorrhage, mainly subdural and subarachnoid [10].

The subarachnoid membrane can disrupt too, causing cerebrospinal fluid overflow to the spaces, adding to the collections growing. In this cases, the ruptured membrane acts like a valve, preventing reflux of the liquid. The clinical condition worsens rapidly, turning it an emergency[10,3].

\section{Retinal hemorrhage}

The retinal vessels are compromised by increased intracranial pressure. This condition can be found in a widespread form, with or without retinosquisis or intravitreal hemorrhage[10].

Despite been an important SBS marker, it can be found in non-traumatic cases, as in ruptured aneurysms, and in accidental traumas, as trafficaccidents[1].

Furthermore, the retinal hemorrhage is reabsorbed in a short time, 7 to 10 days, and it can be small enough to be undetectable. It makes the diagnosis harder [1].

These findings tend to be enhanced when occur other abuse less than 24 hours after the first. However, two repeated episodes aren't able to cause the 
syndrome's clinical manifestations. It takes around ten episodes to appear [8].

Wang et al, 2019, shown the physiological changes in abusive trauma through a simulation with mice. They demonstrated that the time spent to recover consciousness is linked to the abuse intensity. The intensity of the cerebral edema and brain hemorrhage were associated to the injury frequency. Were observed neuronal degeneration and synaptic deficits mainly in the primary motor and sensitive cortex and in the olfactory tubercle. Lastly, neurofunctional changes were found [11].

\section{Diagnosis}

In 1962, SBS was described for the first time. In 1972, was proposed that subdural hematomas (SDH) could be caused by shaking. In 1974, the term "Shaken baby syndrome" was defined as the a set of changes due to abusing[12].

At the same time, based only in medical practice, was observed the association of the syndrome and the presence of SDH. So, it was defined as a syndrome's sign without scientific evidence. Once using it for diagnosing, there was an incidence increase. Then, again without scientific content, it was postulated that SDH and retinal hemorrhage were sufficient to the diagnosis of SBS-"The main Hypothesis". The conviction to diagnose using this only signs was above 94\% between medical doctors[13].

The caregiver was found guilty if he had difficulty to explain the history under investigator's pressure; assume violent shaking and, when he didn't confess, he was declared as a liar[13].

As a result of this historical context, Narang shown that doctors, of all specialties, when facing a SDH and retinal hemorrhage case, tend to define as a SBS diagnose, even though other diagnoses could fit [14].

A great part of the stories told by the caregivers in these cases involved domestic accident, but it was instantly discarded because of the "main hypothesis". Looking for refute this hypothesis, in 1985 the biomechanical proves emerged to simulate the animal movements. So, scientists started to find the inconsistencies in the current postulate[13].

The first biomechanical proves brought many questions, among them the fact that it is impossible for a human to apply similar forces to a car accident at 40 $\mathrm{mph}$ - what can be enough to cause a similar state [13].

Vester et al reviewed biomechanical models to evaluate which type of animal is the best to simulate a child. Lambs are efficient to compare the shaking level and the associated signals and symptoms. Piglets didn't have great trustworthiness because of the anatomical differences, only contributing to analyze single rotation effects in small kinds. The authors affirm that is still necessary to create experiments that move head freely in all directions and without external impacts[5].
On the other hand, using a computational biomechanical model to simulate a 9-year-old child, Jones et al shown that the child's head acceleration depends on neck rigidity, disregarded variable in many studies. It model promises to be more trustworthy simulating using animals [15].

In the same line, Jenny et al performed a biomechanical prove using a anthropomorphic device to simulate a child. They looked for characterize head and neck's cinematics in this type of abuse. They concluded that the spine's force is too important to evaluate the biomechanical results [16].

Nowadays, the diagnose is realized based in a finding triad $(\mathrm{SDH}$, retinal hemorrhage and encephalopathy), besides others broader signs, as apnea, fainting, skull base and long bones fractures and the inconsistent explanation from de parents/caregivers [12]. Since those last sign aren't specific, they cannot draw attention of the less experienced health professionals, delaying the diagnose and management [17].

SDH and encephalopathy can be diagnosed by CT or MRI. The retinal hemorrhage is often diagnosed by fundoscopy[18].

When the of ophthalmic exam is unavailable, the ophthalmic USG can diagnose post-traumatic retinosquisis cases, caused by abusive head trauma [19]. However, it's important to point that large retinal hemorrhage with retinosquisis are not pathognomonic of AHT. Suman et al, 2016 describe two cases that exemplify this affirmation [20].

Högberg et al analyzing the incidence increase of SBS in Sweden concluded that the great cause of this phenomenon isn't because of a real increase in cases, but because of the overdiagnosis, probably caused by a instability in the diagnostic criteria. Although it is not possible to prove this hypothesis, the authors affirm the need of stir up this ethical concerns about syndrome's diagnosis [12].

Dating when the abuse occurred is an extremely important issue in legal investigations that helps delimiting and defining the main suspects [1]. This process is realized based on subdural hematoma's histopathology [21].

As early clinical manifestations appear shortly after the aggression but are usually too soft to draw caregivers attention, there is a difficulty to analyze that content [1]. Besides that, the histomorphological pattern can be changed due to the hematoma fluidity or the surgical drainage act, compromising the analyze [21].

Deilteil et al, sought to create a dating system for subarachnoid and retinal hemorrhage in children to increase the forensics histopathological reports's reliability when it's not possible to evaluate the SDH. A retrospective evaluation of the children who died aging between 0 and 36 months was realized. All of them had gone through forensics histopathological analyze and present subarachnoid and/or retinal hemorrhage $(\mathrm{RH})$. It was concluded that the 
histomorphological changes on HR aren't reliable to dating. On the other side, the presence of siderophages in both types of hemorrhage is robust criteria to dating, once it is found three days after trauma occur [21]. The siderophages's presence makes possible to find hemosiderin in victims retina only until 16,8 mounths after agression, on average [22].

In order to discuss how reliable is the SBS history to justify the triad, Elinder et al, representing the The Swedish Agency for Health Technology Assessment and Assessment of Social Services (SBU), published a systematic review of a case-control and cohort studies with qualitative analysis methods. The first group, that defends the triad as enough evidence to diagnose SBS, was composed by articles that involved more than 10 cases, while the second group represented articles about differential diagnosis that described at least one case. Basing on this evaluation, they conclude that there's no sufficient scientific evidence to evaluate the triad's diagnostic accuracy. Further claim that the scientific evidence even for establish association between the syndrome and the triad are insufficient [7].

Debelle et al severely criticized the SBU's review. According to them, the conclusions departed only from two articles amid the vast researched literature. In their publication, they shown methodological fails, from the configuration to the risks of biases [18].

Bilo further discusses the publication's effects in the courts's judgments of the cases of AHT. They showed that the publication didn't add anything new to the medical knowledge and only exposed the facts allowing even more courts's debates [23].

Rosén et al replied on behalf of the SBU. According to them, any of the articles shown significatives methodological fails. They affirm that the main propose of the review was to encourage other researches to accept the evidence's limitations concerning the syndrome [24].

Even with so many controversies, Tuchtan et al demonstrated, by a reported case, the need of the doctor doesn't hesitate in report suspicious cases, even if there are other probable causes [25].

\section{Imaging Exams}

For an early analyze must be performed a CT, in order to find extra and intra-axial hemorrhages, bone and spine fractures and mass effects. To evaluate cerebral edema and diffuse axonal injury, the CT isn't the best way. Early MRI can be used with CT because it provides a better delineation in cases of cerebral edema, shear injuries and hypoxic-ischemic insult [3].

When associated to a $\mathrm{RH}$ and inconsistent history, the main radiologic finding that raises suspicion of $\mathrm{AHT}$ is the SDH. In some cases, however, it is hard to differ traumatic and non-traumatic causes of SDH. The pontine vein thrombosis indicate a traumatic cause and can be found in some imaging exams- ecogradient, MRI or angiography- and in autopsy [26].

Yilmaz et al described two cases in which was found bilateral multifocal signal losses in pontine veins, what made them propose the idea of being a thrombus formed in compromised pontine veins [26].

The search for differential diagnoses must involve birth trauma. In these cases, the SDH is smaller, located in the posterior fossa or in the tentorium and resolve less than a month of life. When there's hemorrhage it's need to search for history of coagulation disorders. Other conditions are associated to intracranial and retinal hemorrhage are malaria, meningitis and galactosemia, for example. Glutaric aciduria and other bone disorders need to be researched [3][26].

\section{Possible associations in addition to common signs}

Haas-lude et al reported two cases of cerebellar injuries associated to AHT. The authors affirm that, based in that two cases and in the available literature, the pathophysiology of this injuries is associated to severe supratentorial parenchyma lesions. By this form, this type of finding indicate the seriousness of the case [27].

Seider et al reported a case in which was found a macular pseudo-hole linked to AHT detected by an optical coherence tomography [28].

\section{Situations that mimic SBS}

Miller et al described cases in which children presented the triad due to other factors as small falls, spontaneous SDH or related to the delivery and venous sinus thrombosis. The authors point that cases of triad, macrocephaly and increased head circumference can indicate a non-AHT case [29].

There is an hypothesis that AHT can be mimicked by choking/ apparent life-threatening event (ALTE). According to this hypothesis, chocking caused by paroxysmal cough leads to an intrathoracic pressure increase, that is convey to the brain vessels causing $\mathrm{SDH}$ and $\mathrm{RH}$. The hypothesis defends, that the hypoxic period is sufficient to cause brain injuries. Therefore, it could be responsible for the beginning of the triad [30].

Galaznik et al makes a review about the discussions involving this hypothesis [31].

Hansen et al performed a retrospective casecontrol study to teste the reliability of this hypothesis. Childs under two-year-old that had a SDH case were divided between the ones who had or not ALTE. In this study there's no adherence to the hypothesis that ALTE, specifically dysphagic choking, mimic the AHT [30].

\section{Consequences of SBS}


The prognosis of SBS is bad. Between the survivors, only 8 to $36 \%$ have positive results in longterm after treatment [32].

Lind et al performed a retrospective study with child of a rehab clinic diagnosed with AHT between 1996 and 2015. The goal was to evaluate the neurological, cognitive, behavioral and academics consequences in long-term. Several significant results were found, of which we can highlight that of the 47 children analyzed, only seven backed to have a normal life. Two of them had severe neurological impairments. 21 had motor and other 21 visual commitment. In 25 cases there was a behavior change. 38 children still needing rehab. Only 14 ended the conventional curriculum without additional support [33].

In another retrospective study, Antonietti et al evaluate children hospitalized between 2003 and 2013. Among several results found, it became evident that $30 \%$ of the children had neurological changes in the last consult's exam. 12 of the 80 patients developed epilepsy and 19 of them shown behavior changes [34].

On the other hand, Nuño et al demonstrated that older ages, lower income and diagnosis secondary of SBS, as RH, are associated to worse evolution and higher mortality [35].

Bartschat et al reported a case of multicystic encephalomalacia (MEM) after a severe case of AHT. This condition originates in high intensity hypoxicischemic encephalopathy. In these cases occur a replacement of the cerebral parenchyma by cysts, causing a decrease in the differentiation process of the brain cells and consequently a parenchymal atrophy. There's an increase in EME cases due to AHT in the last years [32].

This type of lesion implies economically consequences too. In USA, the medical cost of each child surviving in the first 2,5 post-traumatic years are $\$ 34,750$, on average. On the other hand, the prevention and education expenditures reach $\$ 123,000$. Besides that, in USA, $\$ 39,700$ are spent with children protection service and criminal justice each survivor. Lastly, in New Zeland there's a expense equivalent to $\$ 523,300$ for adaptable equipment, domestic changes and home help service [36].

\section{Risk factors}

Chen et al produced a study in which they used realistic of newborns to analyze parents behavior in children cry. Each of them went through two simulations and in them they could use one of three soothing methods. In some situations, the baby was programmed to cry more (frustration) and in others to cry less (success). They conclude that in situations where the first result was "frustration", $80 \%$ of the parents changed to another method, while only $50 \%$ changed when had success first. This shows why parents tend to shaking repeatedly, once often have succeed in soothing baby in the first episodes [37].

It explains the fact of the biggest incidence age group be similar to the cry curve. Besides this risk factor, financial difficulties, have a male caregiver, be a male child, young parents age and lack of impulse control already shown be associated to a higher incidence [37][38].

Isumi et al, 2017, made a retrospective study using a questionnaire with japanese caregivers of children of 3 to 4 months old. The authors conclude that there's a synergy between unexpected pregnancy and young motherhood and high risk of shaking baby [39].

The same authors, in 2016, had sought to explore if sons of parents who went through adverse situations in their childhood- as physical, sexual or emotional abuse, parents' death, divorce and be homeless- have more risk of suffer choking or shaking. The study was conducted based on the questionnaires answered. The authors affirm don't have found association between both variables [40].

Kelly et al shown in a retrospective case-control study that there's a relationship between the fact of a family being known to child protective services or police at the time of birth and the risk of abusive head trauma. They pointed, however, that this data would add little predictive value to the risk assessment models of this type of trauma in the health services routine [41].

\section{Prevention}

\section{Education and training}

Laurent-Vannier et al applied a questionnaire on health and legal professionals involved on children protection before the start of an AHT training course. The questions were about several aspects of this type of injury. The results shown how little they knew about the syndrome, even if their job is linked to this. $48 \%$ of the doctors weren't aware about the likely complications. 20 to $47 \%$ of the doctors and early childhood professionals didn't know that it was a crime and $20 \%$ to $27 \%$ of them did not know the need to report these cases. It can explain the large amount of undiagnosed cases, showing an important aspect to guide the prevention investments [42] .

Fraser et al, in 2017, described the results of a policy of awareness and training of doctors in Vietnam following an Australian model. The questionnaire data were collected before, six weeks and six months after the training. In the first collect, $28 \%$ had already heard about the syndrome, a much smaller number than the $72,1 \%$ found after six weeks and the $80,3 \%$ found after six months. Recognition of possible damage and of the correct way of control baby's cry has increased after the first collect [17].

If among the professionals involved there is insecurity about AHT, among parents it is higher. Lopes NRL et al, in 2016, using questionnaire shown 
the ignorance of Brazilian parents about the syndrome. Between many worrisome statistics, they demonstrated that $34 \%$ of the Brazilian parents pointed out that use shaking to calm the child. In addition, 34,4\% didn't know that shaking could cause serious consequences for the child. That clarifies the need of prevention strategies aimed at parents [43].

Thinking about this reality, Eismann et al distributed an educational book about the correct ways of soothe the baby, teaching what to do in crying situations. Based on answers of a questionnaire they conclude that this is an applicable alternative that can help others prevention methods [44].

In the same direction, Lopes et al shown, in 2018, that even a quick intervention, as a video and a brochure, is enough to aware parents about the consequences of shaking, ways to soothe baby and about the caregiver's well-being [45].

Mann et al applied a questionnaire in two Ireland's hospitals in which they asked if parents had already heard about SBS and if they want to know more about it. Only half of the parents did. Among them, the most part had this information by social media, journals or friends. On the other hand, almost all parents that had never heard about said that they wanted to know more. Over half of them affirm that the prenatal period is the best to receive this knowledge [46].

In the same line to the previous study, Baar et al described the results after eight years of a national awareness policy about the syndrome on the British Columbia province. The intervention, called PURPLE crying period-nomenclature that indicates aspects of children's crying, as peak of crying, unexpected, resists soothing, pain-like face, long lasting and evening- included all newborns' parents in that region. They made parents aware of the most important points in pre-birth and in an annual awareness campaign. It was noticed an annual decrease of 3.5 and 2.3 cases per 100,000 people in children under 12 and 24 months-old, respectively. The study indicated that there was a reduction of $35 \%$ in the hospitalizations because of AHT there [47].

The same intervention program showed efficiency even when applied on a mostly Spanish-speaking population, in Texas [38]. As well as in the city of Kamagaya, Japan, where the researcher observed the increasing in parents' knowledge after the implementation [6]. In north Caroline, there was a decrease in the number of calls to nursing counseling services after the PURPLE cry period program, however it wasn't possible to show an reduction on AHT cases, according to the authors, because of an intervention's fail [48].

\section{Using technology on prevention}

There's two types of pediatric research based on simulation. The first one analyzes the parents and professional's training. The results indicate the participant's knowledge on technique and skills and relates it to the probable case outcome. The second one evaluate the quality of services provided to the patient, seeking out to identify failures in their conduct [2].

Bechtel et al describe many studies that used that two types of research to evaluate SBS's aspects, from how parents deal with baby's crying to the health professional's skill to recognize a case. The authors conclude that this technique has already shown a big potential and must be explored as a form to reduce the incidence and mitigate the victim's sequelae caused by $\mathrm{AHT}$ [2].

Möller et al performed a research to see if the use of a machine with movement, swaddling and sounds could cause the same effect on soothing a baby of their parents. To analyze the effect's level, it was considered the child's heart rate. They conclude that a similar effect was found in both techniques. The response was greater in younger children. When comparing the heart rate variability, the effect was higher with parents, but, when comparing the heart rate, it was higher in the machine. Because of that, it wasn't possible to clarify which one was more efficient, however, the authors affirm that it could help parents,

Besides the described strategies, Lopes et al reviewed the prevention strategies published between 2005 and 2015. They were divided in managing infants crying, emotional control of caregivers and alerts to the AHT risk factors. Based on this review and in the present article it's possible to analyze the prevention's methods evolution [50].

\section{Effectiveness and difficulties on applying prevention methods}

The World Health Organization recommends that the prevention's programs stablish two main messages: that AHT can cause serious brain injuries/ death and that it's preferable to leave the child crying for a while than shaking [51].

In order to evaluate the effectiveness in passing this messages, Berthold et al applied a questionnaire in Germany's parents. Based on their answers we can perceive that, despite awareness, there's not a significant acceptance of the idea of letting the child cry for more than 5 minutes. Among participants that had already heard about SBS, only 35\% would take the most appropriate conduct, what is not so different of the 33\% among the ones that had never heard about AHT. The authors propose to intensify these messages in the prevention's programs, especially to women. Further, would be interesting, according to them, campaigns deploy that span multiple types of people, as in school, and not only newborns' parents. It demonstrates how hard it is to turn AHT prevention more effectiveness [51].

In 2006, the state of Massachusetts elaborated a law that implemented an awareness program to parents in every birth/center hospital. The program includes nurses' actions from the distribution of 
educational brochures to oral education of parents. The Rideout et al's article highlighted the barriers and the facilitators of this program. Analyzing a questionnaire response data, they defined that leadership support facilitates the application of the program. Otherwise, the factors that hinder is the lack of brochures in another languages, as well as the difficulty of providing oral education in another language. The great barrier pointed for them was the time demand that this process requires, not compatible with the nursing team's routine [52].

\section{Conclusion}

The Shaken baby syndrome, term currently added to the concept of Abusive head trauma, is a disease that have innumerable controversies involving concepts, diagnoses and legal procedures. It prevention is related to population awareness about the correct ways of soothing a child, especially in crying, and about the syndromes' risks. Despite the controversies, new researches need to be done, once it is the main cause of child's brain injury and results in severe consequences for the victims.

\section{Disclosure Statement}

The authors have no conflicts of interest to declare.

\section{References}

1. Vinchon M. Shaken baby syndrome: what certainty do we have? Childs Nerv Syst. 2017 Oct;33(10):17271733.

2. Bechtel K, Bhatnagar A, Auerbach M. Simulationbased research to improve infant health outcomes: Using the infant simulator to prevent infant shaking. Infant Behav Dev. 2019 Aug; 56:101263.

3. Shekdar K. Imaging of Abusive Trauma. Indian J Pediatr. 2016 Jun;83(6):578-88.

4. Hinds T, Shalaby-Rana E, Jackson AM, Khademian Z. Aspects of abuse: abusive head trauma. Curr Probl Pediatr Adolesc Health Care. 2015 Mar;45(3):71-9.

5. van Zandwijk JP, Vester MEM, Bilo RA, van Rijn RR, Loeve AJ. Modeling of inflicted head injury by shaking trauma in children: what can we learn? : Part II: A systematic review of mathematical and physical models. Forensic Sci Med Pathol. 2019 Sep;15(3):423-436.

6. Fujiwara T. Effectiveness of public health practices against shaken baby syndrome/abusive head trauma in Japan. Public Health. 2015 May;129(5):475-82.

7. Elinder G, Eriksson A, Hallberg B, et al. Traumatic shaking: The role of the triad in medical investigations of suspected traumatic shaking. Acta Paediatr. 2018 Sep;107 Suppl 472:3-23.
8. Coats B, Binenbaum G, Smith C, et al. Cyclic Head Rotations Produce Modest Brain Injury in Infant Piglets. J Neurotrauma. 2017 Jan 1;34(1):235-247.

9. Mian M, Shah J, Dalpiaz A, et al. Shaken Baby Syndrome: a review. Fetal Pediatr Pathol. 2015 Jun;34(3):169-75.

10. Wittschieber D, Karger B, Pfeiffer H, Hahnemann ML. Understanding Subdural Collections in Pediatric Abusive Head Trauma. AJNR Am J Neuroradiol. 2019 Mar;40(3):388-395.

11. Wang G, Zhang YP, Gao Z, et al. Pathophysiological and behavioral deficits in developing mice following rotational accelerationdeceleration traumatic brain injury. Dis Model Mech. 2018 Jan 30;11(1). pii: dmm030387.

12.Högberg U, Lampa E, Högberg G, Aspelin P, Serenius F, Thiblin I. Infant abuse diagnosis associated with abusive head trauma criteria: incidence increase due to overdiagnosis? Eur J Public Health. 2018 Aug 1;28(4):641-646.

13.Gabaeff SC. Exploring the controversy in child abuse pediatrics and false accusations of abuse. Leg Med (Tokyo). 2016 Jan;18:90-7.

14. Narang SK, Estrada C, Greenberg S, Lindberg D. Acceptance of Shaken Baby Syndrome and Abusive Head Trauma as Medical Diagnoses. J Pediatr. 2016 Oct:177:273-278.

15. Jones MD, Martin PS, Williams JM, Kemp AM, Theobald P. Development of a computational biomechanical infant model for the investigation of infant head injury by shaking. Med Sci Law. 2015 Oct:55(4):291-9.

16. Jenny CA, Bertocci G, Fukuda T, Rangarajan N, Shams T. Biomechanical Response of the Infant Head to Shaking: An Experimental Investigation. J Neurotrauma. 2017 Apr 15;34(8):1579-1588.

17. Fraser JA, Flemington T, Doan TND, Hoang MTV, Doan TLB, Ha MT. Prevention and recognition of abusive head trauma: training for healthcare professionals in Vietnam. Acta Paediatr. 2017 Oct;106(10):1608-1616.

18. Debelle GD, Maguire S, Watts $P$, Nieto Hernandez R, Kemp AM; Child Protection Standing Committee, Royal College of Paediatrics and Child Health. Abusive head trauma and the triad: a critique on behalf of RCPCH of 'Traumatic shaking: the role of the triad in medical investigations of suspected traumatic shaking'. Arch Dis Child. 2018 Jun;103(6):606-610.

19. Riggs BJ, Trimboli-Heidler C, Spaeder MC, Miller MM, Dean NP, Cohen JS. The Use of Ophthalmic Ultrasonography to Identify Retinal Injuries Associated With Abusive Head Trauma. Ann Emerg Med. 2016 May;67(5):620-4.

20. Shuman MJ, Hutchins KD. Severe Retinal Hemorrhages with Retinoschisis in Infants are Not Pathognomonic for Abusive Head Trauma. J Forensic Sci. 2017 May;62(3):807-811.

21. Delteil C, Kolopp M, Capuani C, et al. Histological dating of subarachnoid hemorrhage and retinal 
hemorrhage in infants. Forensic Sci Int. 2019 Oct;303:109952.

22. Bais B, Karst WA, Kubat B, Verdijk RM. Persistent Retinal Iron in Abusive Head Trauma. J Forensic Sci. $2016 \mathrm{Nov}$;61(6):1693-1696.

23. Bilo RAC. The Swedish Agency for health technology-report about traumatic shaking: much ado about nothing? Forensic Sci Med Pathol. 2018 Dec;14(4):541-544.

24. Rosén M, Lynøe $N$, Elinder $G$, Hallberg B, Sundgren $P$, Eriksson $A$. Shaken baby syndrome and the risk of losing scientific scrutiny. Acta Paediatr. 2017 Dec;106(12):1905-1908.

25. Tuchtan L, Lebreton-Chakour C, Tosello B, Oger M, Piercecchi-Marti MD, Bartoli C. Coexistence of Subdural Hematoma and a Rare Cardiopathy in an Infant: Etiological and French Medicolegal Discussion. J Forensic Sci. 2017 Nov;62(6):1658-1661.

26. Yilmaz U, Körner H, Meyer S, Reith W. Multifocal Signal Loss at Bridging Veins on SusceptibilityWeighted Imaging in Abusive Head Trauma. Clin Neuroradiol. 2015 Jun;25(2):181-5.

27. Haas-Lude K, Roulet-Perez E, Döbler-Neumann M, Groeschel S, Nägele T, Krägeloh-Mann I. Cerebellar lesions in pediatric abusive head trauma. Eur J Paediatr Neurol. 2019 Jul;23(4):604-608.

28. Seider MI, Tran-Viet D, Toth CA. MACULAR PSEUDO-HOLE IN SHAKEN BABY SYNDROME: UNDERSCORING THE UTILITY OF OPTICAL COHERENCE TOMOGRAPHY UNDER ANESTHESIA. Retin Cases Brief Rep. 2016 Summer;10(3):283-5.

29. Miller D, Barnes $P$, Miller M. The significance of macrocephaly or enlarging head circumference in infants with the triad: further evidence of mimics of shaken baby syndrome. Am J Forensic Med Pathol. 2015 Jun;36(2):111-20.

30. Hansen JB, Frazier T, Moffatt M, Zinkus T, Anderst JD. Evaluation of the Hypothesis That Choking/ALTE May Mimic Abusive Head Trauma. Acad Pediatr. 2017 May - Jun;17(4):362-367.

31. Galaznik JG. A response to Mimics of child abuse: Can choking explain abusive head trauma? [35 (2015) 33-37]. J Forensic Leg Med. 2016 Apr;39:46-9.

32. Bartschat S, Richter C, Stiller D, Banschak S. Long-term outcome in a case of shaken baby syndrome. Med Sci Law. 2016 Apr;56(2):147-9.

33. Lind K, Toure H, Brugel D, Meyer P, LaurentVannier A, Chevignard M. Extended follow-up of neurological, cognitive, behavioral and academic outcomes after severe abusive head trauma. Child Abuse Negl. 2016 Jan;51:358-67.

34. Antonietti J, Resseguier N, Dubus JC, et al. The medical and social outcome in 2016 of infants who were victims of shaken baby syndrome between 2005 and 2013. Arch Pediatr. 2019 Jan;26(1):21-29.

35. Nuño M, Pelissier L, Varshneya K, Adamo MA, Drazin D. Outcomes and factors associated with infant abusive head trauma in the US. J Neurosurg Pediatr. 2015 Nov;16(5):515-522.
36. Miller TR, Steinbeigle R, Lawrence BA, et al. Lifetime Cost of Abusive Head Trauma at Ages 0-4, USA. Prev Sci. 2018 Aug;19(6):695-704.

37. Chen M, Barr RG, Miller E, Fairbrother N, Antle A, Brant R. When soothing succeeds: Simulating a risk for repeated shaking in abusive head trauma in infants. Infant Behav Dev. 2019 Aug;56:101246.

38. Duzinski SV, Guevara LM, Barczyk AN, Garcia NM, Cassel JL, Lawson KA. Effectiveness of a Pediatric Abusive Head Trauma Prevention Program Among Spanish-Speaking Mothers. Hisp Health Care Int. 2018 Mar;16(1):5-10.

39. Isumi A, Fujiwara T. Synergistic Effects of Unintended Pregnancy and Young Motherhood on Shaking and Smothering of Infants among Caregivers in Nagoya City, Japan. Front Public Health. 2017 Sep 22:5:245.

40. Isumi A, Fujiwara T. Association of adverse childhood experiences with shaking and smothering behaviors among Japanese caregivers. Child Abuse Negl. 2016 Jul;57:12-20.

41. Kelly P, Thompson JMD, Rungan S, et al. Do data from child protective services and the police enhance modelling of perinatal risk for paediatric abusive head trauma? A retrospectivecase-control study. BMJ Open. 2019 Mar 1;9(3):e024199.

42. Laurent-Vannier A, Chevignard M. Abusive head trauma, healthcare, and childhood professionals' lack of knowledge is detrimental to the child's interest. Arch Pediatr. 2019 May;26(4):199-204.

43.Lopes NRL, Williams LC de A. Avaliação do Conhecimento sobre Trauma Craniano Violento por Pais Brasileiros TT - Brazilian Parents\&\#039; Knowledge on Abusive Head Trauma. Psicol Teor e Pesqui. 2016; 32:e32223-e32223 .

44. Eismann EA, Pearl ES, Theuerling J, Folger AT, Hutton JS, Makoroff K. Feasibility study of the calm baby gently program: An educational baby book intervention on safe practices related to infant crying. Child Abuse Negl. 2019 Mar;89:135-142.

45. Laterza Lopes NR, Górni SM, Mattar VO, de Albuquerque Williams LC. Assessment of a brief intervention with parents to prevent shaken baby syndrome 1. Paideia. 2018; 28:1-9 .

46. Mann AK, Rai B, Sharif F, Vavasseur C. Assessment of parental awareness of the shaken baby syndrome in Ireland. Eur J Pediatr. 2015 Oct;174(10):1339-45.

47. Barr RG, Barr M, Rajabali F, et al. Eight-year outcome of implementation of abusive head trauma prevention. Child Abuse Negl. 2018 Oct;84:106-114.

48. Zolotor AJ, Runyan DK, Shanahan M, et al. Effectiveness of a Statewide Abusive Head Trauma Prevention Program in North Carolina. JAMA Pediatr. 2015 Dec;169(12):1126-31.

49. Möller EL, de Vente W, Rodenburg R. Infant crying and the calming response: Parental versus mechanical soothing using swaddling, sound, and movement. PLoSOne. 2019 Apr 24;14(4):e0214548. 
50. Lopes NRL, Williams LCA. Pediatric Abusive Head Trauma Prevention Initiatives: A Literature Review. Trauma Violence Abuse. 2018 Dec;19(5):555-566.

51. Berthold O, Witt A, Clemens V, Brähler E, Plener $\mathrm{PL}$, Fegert JM. Do we get the message through? Difficulties in the prevention of abusive head trauma.

Eur J Pediatr. 2019 Feb;178(2):139-146.
52. Rideout L. Nurses' Perceptions of Barriers and Facilitators Affecting the Shaken Baby Syndrome Education Initiative: An Exploratory Study of a Massachusetts Public Policy. J Trauma Nurs. 2016 May-Jun;23(3):125-37. 